\title{
The Research of Multipath Query Implementation Based on the City's Public Transportation System
}

\author{
Chong $\mathrm{Xu}^{\mathrm{a}^{*}}$, Yong Shi ${ }^{\mathrm{b}}$ and Ning Tao ${ }^{\mathrm{c}}$
}

School of Information and Control engineering Shenyang Jianzhu University, Shengyang, China

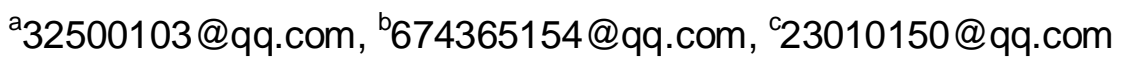

*The corresponding author

Keywords: Many destination; $A^{*}$ algorithm; The optimal path algorithm

\begin{abstract}
In the urban public transport network, usually with a single mesh optimal travel route is the main route query, considering the passenger travel demand, multi-destination travel routes to solve the research problem, through the establishment of the shortest transfer time, minimum transfer times convenient, shortest path query processing module. The algorithms used in this system, $\mathrm{A} *$ algorithm and greed algorithm, and puts forward the optimization of $\mathrm{A} *$ algorithm and the implementation, the algorithm is verified with the data experiment. In the system using the validated to Beijing subway network as an example of multiple destination quickly recommend the feasibility of the optimal route.
\end{abstract}

\section{Introduction}

As an important part of urban traffic network, public transport because of wide coverage, low cost, by using the advantages of the convenient place, multipath recommendation system is designed according to citizens and tourists travel choice habits. In recent years, the domestic transport query system is the most common is the optimal path to a single destination recommendation and transfer plans, due to the continuous development of city size and economic, for example, in a strange city, tourists choose to take public transportation to multiple sites, choose to suit their own line is relatively difficult, how to provide reasonable according to the requirement of the tourists and fast multi-destination bus routes is our study of the problem.

Multi-destination route recommendation system based on MapInfo platform, using spatial database to store data, to deal with multiple sites and line first, and then use Visual c++6.0 Enterprise Edition of MapX secondary development[1], through the establishment of circuit topology structure and the shortest path algorithm, in the $\mathrm{A}^{*}$ algorithm is public transportation network optimization of the shortest path between any two points requirement[2]. All test algorithm using MFC AppWizard, and according to testing results program using MFC AppWizard (exe).

\section{Research Design and Implementation of the Algorithm}

Research Status Related Algorithm. the optimal path algorithm, the shortest path problem has been in the field of such disciplines as computer science, operation research hot topic. Commonly used classic shortest path algorithm with Floyd algorithm[3], A* algorithm, greedy algorithm and diffusion algorithm and Dijkstra algorithm, etc. System for using the above algorithm to solve the shortest path problem, most popular and widely used, the algorithm of the shortest path between two vertices are many, are widely used Dijkstra algorithm to find the shortest path of two points, the time complexity of 0 (iV2), based on the theory of figure has generated, based on the optimization of the data storage structure, trading space for time, priority queue structure optimization of the shortest path. In the process of practical application is also data redundancy, time more computing complexity and large storage space, in order to improve the computation efficiency and save data storage memory, on the basis of the Dijkstra algorithm was improved and optimized, in space, in time to improve the shortest path, improve the efficiency of the algorithm[4]. 
the optimal transfer algorithm. Optimization algorithm in retained BSL lines and site on the basis of the inverted index structure, to transfer information between lines for storage, OPT algorithm is put forward. OPT algorithm is the main idea, first determine the starting site and termination of bus lines, if the same query in the same line, otherwise the route query according to transfer information between lines. BSL with OPT algorithm of route query method between two sites, when requested site more than, will directly affect the query results query sequence between the site.

Greedy Algorithms Implementation. Greedy algorithm is a simple rapid method for solving traveling salesman problem, to local problems from simple to solve the complexity of the optimal solution algorithm, the basic concept can be described as: prioritize, a set of data is processed after the minimum value, with the aim of faster speed to satisfy the selected search method, greedy algorithm is not in the overall consideration in the process of the optimal, but all the problem into several parts, each part of the selection in the range of the whole problem constraints to get the best or optimal solution[5]. Greedy algorithm is the advantage of low time complexity, without considering the whole optimization, the optimum solution in the process of query to find all the exhausted all possible that consume a lot of time, get the overall is not the most optimal solution, but the best approximation of the optimal solution, in the shortest possible time quickly obtain a satisfactory solution.

A* Algorithm To Solve the Optimal Path. Heuristic search in the search process is the feature of joined the heuristic information, $\mathrm{A}^{*}$ algorithm is put forward in the $1960 \mathrm{~s}$, is very important in the heuristic algorithm and efficient algorithms, as one kind of heuristic search algorithm, is the most effective for solving the shortest path in the static network[6], the method of choice by A heuristic function, the current search nodes selection near the least step nodes as the search, when more than one of the least node, the node and connect their side, there are multiple node path, each edge length are given path value this is A graphics in the plane, finally asked out by the cost of the generation algorithm.

Table 1 Analysis of the shortest path data

\begin{tabular}{ccllcll}
\hline $\begin{array}{c}\text { The originating } \\
\text { station-terminal }\end{array}$ & times & $\begin{array}{l}\text { The } \\
\text { shortest } \\
\text { path }\end{array}$ & $\begin{array}{l}\text { The } \\
\text { evaluation } \\
\text { index }\end{array}$ & cost & $\begin{array}{l}\text { Time } \\
\text { consu } \\
\text { ming/ } \\
\text { [min] }\end{array}$ & $\begin{array}{l}\text { The } \\
\text { optimal } \\
\text { route }\end{array}$ \\
\hline $\begin{array}{c}\text { CiqikouStation-Yan } \\
\text { mingyuanStation }\end{array}$ & 3 & 4.53 & 0.4 & 5 & 77 & $5-1-4$ \\
$\begin{array}{c}\text { ShuangjingStation- } \\
\text { ZxiyuanStation } \\
\text { JinsongStation-Taiy } \\
\text { onggongStation }\end{array}$ & 2 & 4.21 & 0.31 & 4 & 56 & $10-4$ \\
\hline
\end{tabular}

Table 2 Analysis of the least transfer data

\begin{tabular}{|c|c|c|c|c|c|c|}
\hline $\begin{array}{l}\text { The originating } \\
\text { station-terminal }\end{array}$ & times & $\begin{array}{l}\text { Minimum } \\
\text { transfer }\end{array}$ & $\begin{array}{l}\text { The } \\
\text { evaluation } \\
\text { index }\end{array}$ & cost & $\begin{array}{l}\text { Time } \\
\text { consum } \\
\text { ing/ } \\
{[\mathrm{min}]}\end{array}$ & $\begin{array}{l}\text { The } \\
\text { optimal } \\
\text { route }\end{array}$ \\
\hline $\begin{array}{l}\text { BeijingStation-Dazo } \\
\text { ngsi Station }\end{array}$ & 2 & 3.73 & 0.9 & 4 & 51 & $2-9$ \\
\hline $\begin{array}{l}\text { DongwuyuanStation- } \\
\text { Yonghegong Station }\end{array}$ & 1 & 5.80 & 0.30 & 3 & 29 & 4 \\
\hline $\begin{array}{l}\text { TiantandongStation- } \\
\text { Pinganli Station }\end{array}$ & 2 & 0.95 & 0.256 & 4 & 40 & $5-6$ \\
\hline
\end{tabular}


$\mathrm{A}^{*}$ algorithm is the core part, choose the path between the start node to the destination node value minimum sequence of nodes to achieve the shortest path search. Is in the process of search it introduced a valuation function node expression for male: $\mathrm{f}(\mathrm{n})=\mathrm{g}(\mathrm{n})+\mathrm{h}(\mathrm{n})$, in which $\mathrm{f}(\mathrm{n})$ is estimated to be worth function, $g(n)$ from the initial position to the search for it represents a node $S$ minimum path to the actual value, to the current node $\mathrm{n}$ to said to the target location of the cost. $\mathrm{H}$ (n) it says, the function design and the selection of the search graph between the two nodes, namely on the current node $n$ is ni A successor to the destination node $G$ path valuation, is whether the optimal path algorithm with heuristic function become $\mathrm{A}^{*}$ algorithm.

\section{Test and Analysis}

In order to further verify the performance of the algorithm, and find out the existing difference between functional requirements and system experiment randomly site circuit testing, set up the demand conditions, the source and multiple destination station name of random input respectively, circuit simulation of the query. Through different ways, according to different preferences for passengers respectively to three time shortest route query, the least change to the route, the shortest distance route query, the results are as follows. The shortest path analysis of data as shown in table 1. At least change to the data analysis as shown in table 2 .

\section{Conclusion}

Multipath in bus network provide people with better travel information query system and scheme. In the later study, but also give full consideration to the city subway structure characteristics, and the route parameters used for the shortest path algorithm design and optimization, the basic query functions in satisfying users can adapt to the environment and the line transportation, improve the efficiency of people travel in practical application.

\section{Reference}

[1] LIXiaopeng, YU BinandLI Ya-min. Shortest path search algorithm based on MapX. Computer engineering and design,2009, 30(22).

[2] ZhenFeng, JiaLi, JingLiandYFCao. The algorithm of shortest path problems in complex networks research [J]. Automation technology and applications, 2010, 29 (3):32-35.(In Chinese)

[3] XyWangandQhLi.The shortest path analysis based on GIS [J]. Journal of logistics engineering and management, 2012, 6 (34): 1674-4993.(In Chinese)

[4] ZWJianandJfFeng. City bus transfer model based on GIS and implementation [J]. Journal of urban surveying, 2014, 6 (3): 42-47.(In Chinese)

[5] A. Using Decision Tree Classifiers in Source Code Analysis to Recognize Algorithms: An Experiment with Sorting Algorithms.2011,54(11).

[6] Nivoliers. L-system specification of knot-insertion rules for non-uniform B-spline subdivision. [J].Computer Aided Geometric Design,2011,29(2). 